\title{
ÚLCERAS POR PRESSÃO, PREVENÇÃO PRIMÁRIA E EDUCAÇÃO: REVISÃO INTEGRATIVA DE ESTUDOS
}

\author{
L. M. F.Fialho ${ }^{1^{*}}$, M. V. Baron ${ }^{2}$,C. Brandenburg ${ }^{3}$, J. R. Santana ${ }^{4}$, J. Koepp ${ }^{5}$ \\ ${ }^{1}$ Universidade Estadual do Ceará- ${ }^{2,5}$ Universidade de Santa Cruz do Sul $-{ }^{3,4}$ Universidade Federal do Ceará \\ lia_fialho@yahoo.com.br
}

\section{RESUMO}

Realizar uma revisão de literatura integrativa de artigos publicados (2010-2013) em bases de dados científicas. O escopo é verificar quais medidas de prevenção primária e ações educativas são disseminadas para evitar e tratar Úlceras por Pressão (UP) ao desenvolver uma descrição do conteúdo dos artigos científicos, diretamente relacionados a temática em tela, que foram indexados à Biblioteca Virtual de Saúde (BVS). Foram selecionados os 13 artigos disponíveis gratuitamente na íntegra, que correspondiam ao tema de investigação para compor a fonte da investigação. Constatou-se, no que concerne ao emprego de medidas preventivas, que, enquanto algumas exprimem efeitos positivos comprovados em estudos com bom nível de evidência, outras não apontam efeitos suficientemente comprovados como benéficos ou eficazes. Em relação às medidas educativas, os estudos convergem ao indicar resultados positivos, pois a educação das equipes foi considerada um dos pilares de sucesso no tratamento de UP.

PALAVRAS-CHAVE: Úlceras por pressão. Prevenção primária. Educação.

\section{PRESSURE ULCERS, PRIMARY PREVENTION AND EDUCATION: AN INTEGRATIVE REVIEW OF STUDIES}

\section{ABSTRACT}

To do an integrative literature review of the published articles (2010-2013) in scientific databases. The scope is to verify which primary prevention measures and educative actions are disseminated to avoid and treat pressure ulcers (PU), when developing a description of the content of scientific articles directly related to this theme, that were indexed to the Science Virtual Library. The 13 full articles available for free that corresponded to the investigation theme were selected to compose the investigation source. It was verified, concerning the employment of preventive measures, that, while some express positive effects attested in studies with a good level of evidence, others do not point to effects sufficiently attested as neither beneficial nor efficient. In relation to educative measures, the studies converge to indicate positive results. The education of the groups was considered one of the pillars of success in the PU treatment.

KEY-WORDS: Pressure ulcers, Primary prevention, Education. 


\section{INTRODUÇÃO}

A úlcera por pressão (UP) pode ser definida como "uma área localizada de necrose celular que tende a desenvolver-se quando os tecidos moles são comprimidos entre uma proeminência óssea e uma superfície dura por um período prolongado de tempo"(Miranda et al., 2012, p. 8-9).

A pressão, a fricção e o cisalhamento são os principais fatores extrínsecos responsáveis pelo aparecimento da UP, e a diminuição da mobilidade, a incontinência urinária e fecal, a idade avançada, o défice nutricional, a diminuição do nível de consciência e a presença de comorbidades crônicas são os principais elementos intrínsecos que contribuem para o seu aparecimento. Uma pessoa pode desenvolver uma UP em apenas duas horas com a mesma postura, e a permanência em posição deitada e/ou sentada por um determinado período, normalmente, enseja estas lesões localizadas na pele, podendo estender o processo destrutivo ao músculo e osso (Baron, Carneiro, Fialho \& Santana, 2012).

A evolução da úlcera ocorre pela infecção bacteriana, o que conduz a destruição do tecido infectado rapidamente, podendo provocar septicemia e contribuir diretamente para a morte do paciente (Hess, 2002). Estima-se que no Brasil a prevalência de UP em pacientes internados varia de $3,5 \%$ a $27 \%$ e pode chegar a $50 \%$ dos pacientes em acompanhamento domiciliar (Leite, Sanches\& Santiago, 2010).

Há consenso entre estudiosos acerca do fato de que a maior parte das UP pode ser prevenida com a adoção de medidas, reduzindo o risco de desenvolver as UP em 25 a $50 \%$ (Dardengoet al., 2006; Rocha,Miranda\&Andrade, 2006). Os custos relacionados com a prevenção das UP é menos dispendioso no que se refere aos aspectos psíquicos e sociais relacionados ao sofrimento do paciente e da família (Paranhos, 2003).

A prevenção primária, portanto, deve ser considerada prioridade no cuidado da pessoa em relação às UP, e requer uma abordagem sistemática, que se inicia com avaliação individualizada e prossegue com a adoção de medidas preventivas que envolvem toda a equipe de saúde (Dardengoet al., 2006). A prevenção primária ancorada em programas educacionais para a prevenção de lesões causadas pela pressão deve estar à disposição de todos os níveis de prestadores de serviços de saúde, pacientes e seus componentes familiares ou atendentes. As equipes de saúde devem privilegiar o aspecto educacional no atendimento, que dará suporte às práticas adequadas de prevenção e profilaxia, assim, evitando o problema (Epuap-Npuap, 2009).

Com suporte no exposto, questiona-se: que medidas de prevenção primária e de educação os profissionais de saúde utilizam para evitar a UP, e que são veiculadas em pesquisas científicas recentes (2010 a 2013)? O objetivo deste estudo, com efeito, é realizar uma revisão de literatura integrativa de artigos publicados (2010-2013) em bases de dados científicas. O escopo é verificar quais medidas de prevenção primária e ações educativas são disseminadas para evitar e tratar UP, ao se desenvolver uma análise crítica do conteúdo dos artigos científicos, diretamente relacionados à mencionada temática, indexados à Biblioteca Virtual de Saúde (BVS).

A relevância do tema reside no fato de que ocorrem altos índices de mortalidade e morbidade decorrentes das UP no mundo todo, o que faz da prevenção e tratamento da UP um desafio atual. Ao tempo que os cuidados consistem na abordagem multidisciplinar, fundamentada em medidas preventivas primárias e educativas simples e viáveis, o trato com 
as UP por vezes é negligenciado ou relegado a segundo plano de importância, ganhando proporções maiores que chegam a ocasionar óbitos por infecção (Dardengoet al. 2006; Rocha et al., 2006).

\section{Delineamento Metodológico}

A revisão da literatura é parte imprescindível da investigação e compreende localizar, averiguar, condensar e interpretar a investigação prévia (revistas cientificas, livros, atas de congressos, resumos etc.) relacionada com a área de estudo afim; é, então, uma análise pormenorizada de trabalhos publicados sobre o tema investigado. A revisão da literatura é fundamental para definir o problema da pesquisa e, também, para obter uma ideia certa sobre o estado d'arte de determinado tema, as suas lacunas e o contributo da investigação para incrementar o conhecimento (Bento, 2012).

A prática da revisão bibliográfica baseada em evidências trata-se de uma ação orientada, onde há uma integração ativa do conhecimento tácito e intencional, associada a processos que asseguram sua qualidade. Possibilitando, consecutivamente, o uso prático das informações sintetizadas pelo pesquisador em seu trabalho diário(Galvão,Sawada\&Mendes, 2003). As recentes iniciativas no campo da prática baseada em evidências aumentaram a necessidade e a produção de todos os tipos de revisão literária - revisão integrativa, revisão sistemática, meta-análise e revisão qualitativa - que tem contribuído para métodos mais sistemáticos e rigorosos (Whittemore \& Knafl, 2005).

Para o desenvolvimento desta revisão integrativa, optou-se pela proposição de Ganong (1987), percorrendo as seguintes etapas: 1) identificação da pergunta norteadora, que se fundamenta na elaboração de um problema de maneira definida e concisa, sucedida da busca por descritores ou palavras-chave; 2) seleção da amostragem e fixação de critérios de inclusão e exclusão, estabelecendo clareza, propriedade e confiabilidade na seleção; 3) categorização dos estudos - extração das informações dos artigos, sintetização e organização destes; 4) avaliação dos estudos e análise crítica dos dados; 5) discussão e explanação dos resultados - estes são precípuos e confrontados e fundamentados com o conhecimento teórico e avaliados quanto a sua utilidade; 6) exposição da revisão integrativa por intermédio da síntese das evidências encontradas de cada artigo de forma sistematizada.

Conforme Melnyk e Fineout-Overholt (2005), a apreciação do nível de evidência foi categorizada em sete níveis: 1- as evidências provêm revisão sistemática, metanálise ou de diretrizes clínicas oriundas de revisões sistemáticas de ensaios clínicos randomizados e controlados; 2- evidências de pelo menos um ensaio clínico randomizado controlado; 3evidências derivadas de ensaios clínicos bem delineados sem aleatorização; 4- evidências oriundas de estudo de coorte e de caso-controle bem delineados; 5 - evidências apresentadas de revisão sistemática, de estudos descritivos e qualitativos; 6- evidências provenientes de um único estudo descritivo ou qualitativo; 7- evidências derivadas da opinião de autoridades e/ou parecer de comissão de especialistas.

No que tange ao levantamento bibliográfico, foi realizada pesquisa na BVS (Biblioteca Virtual em Saúde) que integra fontes eletrônicas de informação de Ciências em Saúde em Geral de bases de dados como: LILACS (Literatura Latino-Americana e do Caribe em Ciências da Saúde), IBECS (Índice Bibliográfico Espanhol de Ciências da Saúde), MEDLINE (Literatura Internacional em Ciências da Saúde), Biblioteca Cochrane, SciELO (Scientific Eletronic Library 
Online), e da base de dados do Portal de Evidências, que integra revisões sistemáticas, ensaios clínicos, sumários de evidência, avaliações econômicas em saúde e avaliações de tecnologias em saúde. O descritor utilizado na busca foi "úlcera por pressão", associado aos termos "prevenção primária" e "educação".

A pesquisa utilizou os seguintes critérios de inclusão: artigos que abordem o tema úlceras por pressão, prevenção primária e educação, indexados nas bases de dados supracitadas, publicados no período de janeiro de 2010 a setembro de 2013, com textos disponíveis, sem custo de acesso, na íntegra, por meio eletrônico nos idiomas Português, Inglês ou Espanhol. Como critérios de exclusão, foram desconsiderados artigos não disponíveis na íntegra ou que não privilegiavam o tema investigado, ainda que possuíssem os descritores de consulta.

Para sumariar e organizar as informações dos achados dos estudos e formar banco de dados incluídos nesta revisão integrativa, elaborou-se um quadro sumário cobrindo os itens: título original do artigo, identificação dos autores, ano de publicação, base de dados publicada, nome do periódico, nível de evidência do artigo (Melnyk \& Fineout-Overholt, 2005), objetivos do estudo, delineamento metodológico, resultados, conclusões e recomendações. Os dados foram categorizados e discutidos segundo os objetivos da revisão integrativa: verificar que medidas de prevenção primária e ações educativas são disseminadas na BVS para evitar e tratar o aparecimento de UP.

\section{Resultados}

Foram encontrados 140 artigos com o descritor "úlcera por pressão", associado aos termos "prevenção primária" ou "educação". Destes, 127 foram descartados - 42 porque não atendiam diretamente ao tema da pesquisa, 29 não possuíam acesso ao resumo ou ao texto, sem ônus, 56 disponibilizaram apenas os resumos para leitura sem custo. Após efetuada a exclusão segundo critérios previamente definidos, sobraram 13 escritos, selecionados como fonte de pesquisa desta revisão. Do total de peças investigadas, dez pertenciam à base de dados MEDLINE, 1 à LILACS, 1 à IBECS e 1 à base SciELO. Os resultados dos artigos selecionados foram sumariados para melhor apresentação no Quadro 1.

\section{Artigo 01 - Heel pressure ulcers: purple heel and deep tissue injury}

Autor (es): Salcido, Lee \&Ahn

Ano de publicação e base de dados: 2011 (MEDLINE)

Periódico: Advances in skin \& wound care

Nível de evidência: 5

Objetivos: auxiliar a interpretação de fatores que contribuem para possíveis UP em calcanhar e aplicar os conhecimentos adquiridos para identificar fatores de risco, prevenção e tratamento da UP, destinguindo de lesão profunda de tecidos e de calcanhar roxo nos locais de atendimento ao paciente.

Delineamento: estudo de revisão.

Resultados, conclusões e recomendações: A prevenção eficaz de UP no calcanhar envolve uma abordagem multifacetada, incluindo identificação de riscos, avaliação de comorbidades e implementação de dispositivos de redistribuição de pressão precocemente. Embora o calcanhar roxo não seja concretamente reconhecido como síndrome, alterações súbitas na tonalidade da pele indica ulceração iminente e progressão da isquemia e necrose.Em contraste com a LPT, não é 
evidente que o calcanhar roxo seja exclusivamente consequência de pressão e friç̧ão; pode ser compátivel com outras patologias.

Artigo02 - Pressure ulcer risk assessment and prevention: a systematic comparative effectiveness review.

Autor (es): Chou et al.

Ano de publicação e base de dados: 2013 (MEDLINE)

Periódico: Annalsof Internal Medicine

Nível de evidência: 1

Objetivos: revisar a utilidade clínica de instrumentos de avaliação de risco de UP e a eficácia de intervenções preventivas em pessoas com maior risco.

Delineamento: estudo de revisão sistemática. Pesquisa em bases de dados MEDLINE (1946 a 2012), CINAHL, Biblioteca Cochrane, registros de ensaios clínicos e listas de referências.

Resultados, conclusões e recomendações: superfícies de apoio estático sofisticados são mais eficazes do que colchões-padrão para prevenir UP nas populações de maior risco. Evidências sobre a eficácia da suplementação nutricional, reposicionamento e intervenções de cuidados da pele, bem como o cuidado usual era limitado e tinha falhas metodológicas, impedindo fortes conclusões.

Artigo 03 - How to reduce hospital-acquired pressure ulcers on a neuroscience unit with a skin and Wound assessment team.

Autor (es): Mcguinness et al.

Ano de publicação e base de dados: 2012 (MEDLINE)

Periódico: Surgical Neurology International

Nível de evidência: 6

Objetivos: controlar e prevenir UP adquirida em hospital por meio de equipe de Enfermagem especializada, sem aumentar as despesas com pessoal, reduzindo/evitando custos.

Delineamento: estudo descritivo, quantitativo e transversal. O cuidado foi instituído em unidade de neurociências (Neurologia / Neurocirurgia) unidade de UTI com 14 leitos e unidade com 18 leitos no andar no período de três anos (2008 a 2011). Cuidados para reduzir UP nas instituições hospitalares em 2008: (1) reposicionamento a cada 1-2 h, (2) camas especiais, e (3) equipe de avaliação da pele e feridas (SWAT), que incluía um ou dois especialistas enfermeiros/auxiliares de enfermagem que faziam visitas a todos os pacientes da unidade, pelo menos uma vez por semana. Em 2010, novas medidas incluíram: (1) a adição de oito camas Stryker (especiais para pacientes de alto risco), (2) botas protetoras de calcanhar para aliviar a pressão e (3) todas as novas equipes foram educadas pela equipe SWAT.

Resultados, conclusões e recomendações: no primeiro ano (2009), alcançou-se uma redução de $48 \%$ de UP na Unidade de Terapia Intensiva (UTI), e redução de 59\% no andar. Estas reduções foram melhoradas em 2010 (UTI redução de 57\% e no andar redução de 65\%) e 2011 (redução de $61 \%$ na UTI e redução $82 \%$ no andar). A introdução de equipes especialistas para avaliar rotineiramente a pele de pacientes com doença aguda, seja em unidade de Neurologia, Neurocirurgia, cardiovascular, reabilitação ou outras unidades médicas / pós-operatório, parece ser um método eficaz para reduzir UP. Além disso, a mesma equipe de enfermagem (sem novas contratações, sem horas extras, e sem aumentar os custos) pode compô-las. Pacientes se beneficiam com a redução da UP adquirida no hospital, reduzindo a morbidade/mortalidade, desfrutando de hospitalizações potencialmente menores e incorrendo em custos mais baixos.

Artigo 4 - Preventing pressure ulcers in long-term care: a cost-effectiveness analysis. 


\author{
Autor (es): Pham et al. \\ Ano de publicação e base de dados: 2011 (MEDLINE) \\ Periódico: JAMA Internal Medicine \\ Nível de evidência: 2
}

Objetivos: avaliar o custo-efetividade de estratégias baseadas em evidências para melhorar as práticas de prevenção em instalações de cuidados de longo prazo.

Delineamento: estudo de revisão. Dados derivados de uma coorte de base populacional em Ontário, Canadá (de maio de 2004 a novembro de 2007), pesquisa telefônica para saber a prática de prevenção atual, bancos de dados administrativos (Appendix) e revisões da literatura, no período 2008 a 2009 pelo Comitê Consultivo de Tecnologia da Saúde de Ontário.

Resultados, conclusões e recomendações: foram consideradas as seguintes estratégias baseadas em evidências para melhorar as práticas de prevenção em curso. Estratégia 1: substituir todos os colchões padrão em instalações de cuidados de longo prazo por colchões de redistribuição de pressão (provas de cinco ensaios clínicos randomizados (ECR). Estratégia 2: fornecer suplementos nutricionais orais diários para residentes de alto risco com perda de peso recente (4 ECR). Estratégia 3: aplicar emoliente diário para a pele seca dos residentes de alto risco (1 ECR, 380 participantes). Estratégia 4: substituir água e sabão por um limpador de espuma para os cuidados de incontinência urinária e intestinal (1 ECR, 132 participantes). Cada estratégia de intervenção foi observada isoladamente. $\mathrm{O}$ estudo mostrou que a qualidade do colchão associada à estratégia de melhoria é susceptível de melhorar a saúde e reduzir os custos. Conforme revisões atuais, suplementos nutricionais orais estão associados a efeito de prevenção significativo, mas pequeno, e foi mostrado que a estratégia associada não é rentável, mas pode haver amplos benefícios para a saúde, por exemplo, apoiando outras estratégias e revertendo a perda de peso não intencional e a redução de infecções que não foram analisadas neste estudo. Em relação à estratégia 3 , dada a evidência clínica disponível, foi mostrado que a estratégia associada pode ser rentável, mas conclusão mais firme não é possível sem dados clínicos adicionais. Em relação à estratégia 4, mostrou que a estratégia associada pode melhorar a saúde, economizar tempo pessoal e reduzir os custos. Dados clínicos adicionais, no entanto, são necessários.

Artigo 05 - Úlceras por presión en personas con lesión medular: conocimiento de familiares y cuidadores.

Autor (es): Figueiredo et al.

Ano de publicação e base de dados: 2010 (LILACS)

Periódico: Avances enenfermería

Nível de evidência: 6

Objetivos: avaliar o conhecimento dos fatores de risco e prevenção de UP do ponto de vista de familiares e cuidadores de pacientes com lesão medular em casa.

Delineamento: estudo descritivo, quantitativo, transversal. Questionário adaptado com 36 intervenções com variáveis: medidas de tratamento, prevenção e fatores de risco para UP aplicado para familiares e cuidadores, de agosto a dezembro de 2008 na cidade de Fortaleza, Brasil.

Resultados, conclusões e recomendações: o estudo mostra que as famílias e cuidadores têm bom conhecimento e estão preparados para o cuidado, no entanto, a alta incidência de erros nas medidas de prevenção das UP mostra que eles não têm conhecimento adequado sobre tais medidas. A pesquisa indica que avaliar o conhecimento de familiares e cuidadores é essencial para implantar ações corretivas para cuidar adequadamente pessoas com lesão medular.

Artigo 06 - Decubitus ulcers: pathophysiology and primary prevention.

Autor (es): Anders et al. 
Ano de publicação e base de dados: 2010 (MEDLINE)

Periódico: Deutsches Arzteblatt International

Nível de evidência: 5

Objetivos: melhorar a compreensão das causas da UP e os mecanismos pelos quais elas se desenvolvem, os fatores de risco e os pacientes que estão em risco, familiarizar as medidas preventivas específicas e capacitar para informar e aconselhar os pacientes e suas famílias sobre UP.

Delineamento: Estudo de revisão realizado no período de 2004 a 2009, na Alemanha.

Resultados, conclusões e recomendações: pacientes idosos, com múltiplas morbidades associadas à imobilidade são de alto risco para o desenvolvimento de UP, assim como os pacientes paraplégicos. Os fatores de risco que promovem o desenvolvimento de UP devem ser minimizados tanto quanto possível. A aplicação e eficácia das medidas de prevenção devem ser reavaliadas e documentadas. Fatores de risco para o desenvolvimento de UP devem ser avaliados no primeiro contato do médico com o paciente imóvel, ou logo que a condição do paciente se deteriora, o que é um prerrequisito para a prevenção oportuna. Uma vez que os riscos foram avaliados, devem ser tomadas medidas terapêuticas com base no perfil de risco individual do paciente, com ênfase no encorajamento ativo do movimento e alívio passivo da pressão por mudanças frequentes de posição.

Artigo07 - Preventing in-facility pressure ulcers as a patient safety strategy: a systematic review.

Autor (es):Sullivan \& Schoelles

Ano de publicação e base de dados: 2013 (MEDLINE)

Periódico: Annalsof Internal Medicine

Nível de evidência: 1

Objetivos: rever estratégias para a prevenção de UP e analisar a importância dos aspectos contextuais de programas que visam a reduzir as UP adquiridas na internação.

Delineamento: Estudo de revisão sistemática. Revisão de estudos de 2000 a 2012 nos EUA.

Resultados, conclusões e recomendações: as conclusões de 26 estudos de implementação de iniciativas para evitar UP em adultos em contexto de cuidado de pacientes agudos e internados em longo prazo (força moderada de evidência) sugeriram que a integração de vários componentes na melhoria dos processos de atendimento reduziram as taxas de UP. Componentes-chave incluem a simplicação e a padronizaçao de intervenções específicas para prevenir UP e a documentação destas, o envolvimento de equipes multidisciplinares e de liderança, e a formação de especialistas designados para avaliação da pele, cursos de educação de funcionários, auditorias e feedback.

Artigo08 - Efectividad de una intervención formativa en prevención de úlceras por presión en una unidad de cuidados intensivos quirúrgica: un estudio cuasi experimental.

Autor (es): Picatoste et al.

Ano de publicação e base de dados:2012 (SciELO)

Periódico: Gerokomos

Nível de evidência: 6

Objetivos: avaliar o impacto de uma intervenção educativa sobre prevenção de UP por meio da estimativa da incidência e gravidade das lesões nos períodos antes e após a intervenção em uma unidade de cuidados intensivos cirúrgica.

Delineamento: estudo quase experimental com pré e pós-controles em UTI cirúrgica em Madrid, no período de fevereiro de 2009 a agosto de 2010. Participaram 247 pacientes no período préintervenção educativa e 200 na pós-intervenção educativa. 
Resultados, conclusões e recomendações: Os resultados confirmam a eficácia das intervenções educativas na redução da incidência de UP. Embora não sejam dados estatisticamente significativos, os pesquisadores acreditam que é uma diminuição clinicamente importante, porque envolve uma melhoria considerável da qualidade de vida do paciente em UTI. Outro ponto relevante é o aumento dos registros de enfermagem em 46,6\%. Os resultados deste estudo confirmam a eficácia das ações educativas em UTI cirúrgica na redução da incidência de UP, incidência de UP fase I, e promoveu melhoria nos registros de enfermagem.

Artigo09 - Randomized multilevel intervention to improve outcomes of residents in nursing homes in need of improvement.

Autor (es): Rantz et al.

Ano de publicação e base de dados: 2012 (MEDLINE)

Periódico: Journal of the American Medical Directors Association

Nível de evidência: 2

Objetivos: testar uma intervenção experimental focada na capacidade organizacional para criar e sustentar a melhoria na qualidade do atendimento de residentes em lares de idosos.

Delineamento: ensaio clínico randomizado, realizado no Missouri. O grupo intervenção ( $n=29$ lares de idosos) recebeu intervenção experimental multinível por dois anos e foram abordados: (1) uso de métodos para melhoria da qualidade, (2) uso da equipe e processos de grupo para melhorar o atendimento direto e a tomada de decisão, (3) foco na realização de cuidados básicos e (4) manter a enfermagem mais consistente e liderança administrativa comprometida com a comunicação e a participação ativa dos funcionários na tomada de decisões. O grupo-controle ( $\mathrm{n}=$ 29 lares de idosos) recebeu informações mensais sobre o envelhecimento e avaliação física dos idosos.

Resultados, conclusões e recomendações: a incontinência urinária e intestinal, perda de peso, UP e diminuição da Atividade de Vida Diária(AVD) foram selecionadas como as medidas de resultados para este estudo.A intervenção melhorou a qualidade do atendimento $(p=0,02)$, houve melhoras em UP $(p=0,05)$ e, na diminuição da perda de peso $(p=0,05)$, não foram encontrados níveis de significância na incontinência e na AVD. A permanência de funcionários, condições de organização do trabalho, recursos humanos, composição das equipes e a maior parte dos custos não foram afetados pela intervenção. A rotatividade da liderança foi surpreendentemente excessiva em ambos os grupos - intervenção e controle. Conforme os pesquisadores, a intervenção abrangente de vários níveis não foi suficiente para resultar em melhorias organizacionais esperadas, na eficiência de custos, permanência de funcionários e, nas condições de trabalho da organização; sugere uma intervenção com gestão da educação mais intensiva e treinamento em combinação com consultas mensais no local, com foco em melhoria de cuidados e os processos da equipe.

Artigo10 - Improving identification and documentation of pressure ulcers at an urban academic hospital.

Autor (es): Dahlstromet al.

Ano de publicação e base de dados: 2011 (MEDLINE)

Periódico: Joint Commission Journal on Quality and Patient Safety

Nível de evidência: 5

Objetivos: avaliar o impacto de uma campanha de multicomponentes sobre a qualidade da documentação de UP como um método de quantificação da melhoria na prevenção e tratamento.

Delineamento: Revisão retrospectiva. Dados coletados com base em uma campanha realizada na University of Chicago Medical Center entre 2007 e 2009. A campanha incluiu: (1) materiais de referência sobre UP, (2) novos modelos de documentação, (3) educação pessoal e (4) e 
substituição de todos os colchões do hospital.

Resultados, conclusões e recomendações: a campanha aumentou significativamente a documentação completa de UP pelos enfermeiros de $27 \%$ para $55 \%$ (OR 3,68, p = 0,001, IC 95\%: $1,68-8,08)$. Os pesquisadores concluíram que houve melhora na documentação de UP por enfermeiros e médicos e foram utilizados descritores como: estágio, tamanho ou localização da UP. Porém estas melhorias foram de curta duração em virtude da implementação de registros médicos eletrônicos (EMR) para anotações de enfermagem. O percentual de documentação da UP pelos enfermeiros diminuiu após a implementação do EMR e a documentação por médicos caiu para $0 \%$ e não se recuperou. Os autores enfatizam a importância de intervenções frequentes e repetidas para manter o sucesso e melhoria da qualidade na prevenção e tratamento de UP.

Artigo11 - Factors impacting the physical function of older adults in Korean long-term care hospitals.

Autor (es): Lee, Kim \& Cho

Ano de publicação e base de dados:2011 (MEDLINE)

Periódico: Journal of Korean Academy of Nursing

Nível de evidência: 6

Objetivos: examinar as AVDs dos idosos internados em hospitais coreanos de cuidados de longo prazo e explorar fatores do paciente e organizacionais e seus impactos.

Delineamento: estudo de revisão. Análise secundária do conjunto de dados mínimo coreano (KMDS) de pacientes $(n=14.369)$, onde os perfis de idosos $(N=358)$ foram analisados a partir da revisão do seguro de saúde e serviço de avaliação realizado de janeiro a julho de 2008.

Resultados, conclusões e recomendações: dos pacientes, $45,4 \%$ tiveram pontuação inicial da AVD de 31 a 40, onde a pontuação de 40 indica que o paciente era totalmente dependente. Os autores concluíram que, para manter e melhorar a AVD de idosos internados em hospitais de cuidados de longo prazo, deve-se desenvolver estratégias para prevenir a incontinência urinária e fecal, UP, tubos ou cateteres desnecessários, oferecendo fisioterapia adequada. Sugerem que estudos adicionais devem incluir informações mais detalhadas sobre a equipe de enfermagem, incluindo horas de atendimento direto, nível de educação e volume de trabalho.

Artigo12 - The relationship of pressure ulcers, race, and socioeconomic conditions after spinal cord injury.

Autor (es): Saunders, Krause\& Reed

Ano de publicação e base de dados: 2010 (MEDLINE)

Periódico: The Journal of Spinal Cord Medicine

Nível de evidência: 4

Objetivos: identificar os fatores de riscos associados com UP após a lesão medular, examinando etnia e indicadores de nível socioeconômico medido pela renda e educação.

Delineamento: estudo de coorte. Responderam a pesquisa 1466 estadunidenses, entre brancos e africanos adultos, com pelo menos um ano de lesão da medula espinhal (LME) por trauma. Foram identificados por meio de registros de atendimento em hospital de reabilitação dos EUA.

Resultados, conclusões e recomendações: a etnia afro-americana foi significativamente associada a ter uma UP com necessidade de cirurgia para repará-la. Renda mais baixa foi associada com o aumento de chances de desenvolver UP; e nível mais baixo de educação foi associado com o aumento de chances de ter uma úlcera atual. Especificamente, a falta de recursos, tanto financeiros como educacionais, está associada a piores resultados para úlceras. E estes resultados podem ser usados por prestadores de serviço e por responsáveis políticos, ao considerar estratégias de prevenção e intervenção para pessoas com LME. 


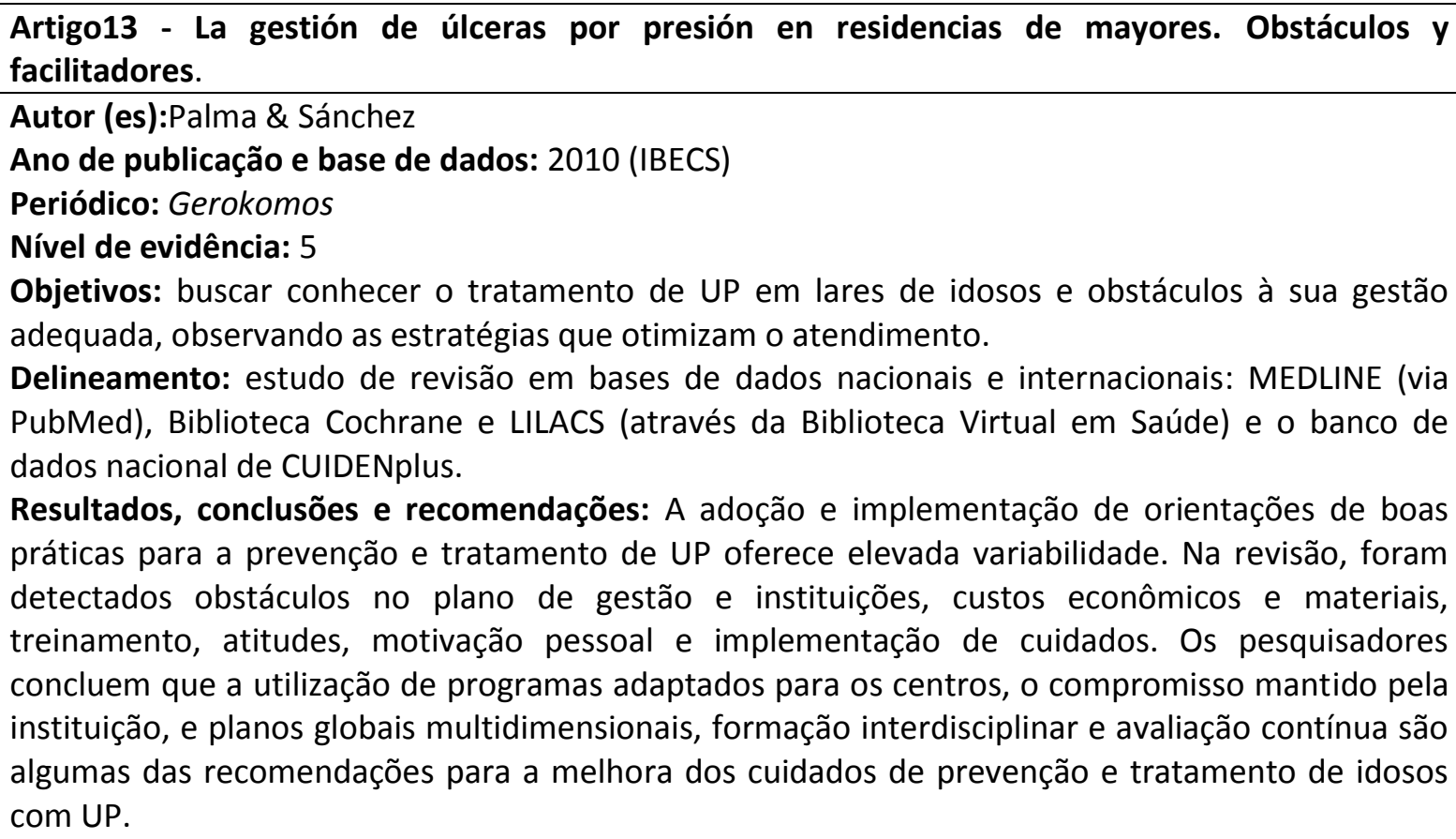

Resultados, conclusões e recomendações: $A$ adoção e implementação de orientações de boas práticas para a prevenção e tratamento de UP oferece elevada variabilidade. Na revisão, foram detectados obstáculos no plano de gestão e instituições, custos econômicos e materiais, treinamento, atitudes, motivação pessoal e implementação de cuidados. Os pesquisadores concluem que a utilização de programas adaptados para os centros, o compromisso mantido pela instituição, e planos globais multidimensionais, formação interdisciplinar e avaliação contínua são algumas das recomendações para a melhora dos cuidados de prevenção e tratamento de idosos com UP.

Quadro 1 - Sumário dos artigos de úlcera por pressão veiculados na BVS.

Em relação ao delineamento metodológico no tocante ao nível de evidencia, constatou-se que: dois dos estudos expressam nível de evidência 1, com forte recomendação na escolha, configurando-se excelentes os níveis de evidência para recomendar rotineiramente a conduta; dois exprimem nível de evidência 2, que recomenda a ação; relatam evidências importantes no desfecho, com a conclusão de que há benefício na escolha da ação em relação aos riscos do dano; um indicou nível de evidência 4, pois encontra mínimas evidências satisfatórias na análise dos desfechos, ele conclui que os benefícios e os riscos do procedimento não justificam a generalização da recomendação e não demonstra posicionamento contra ou a favor da ação de prevenção e tratamento; quatro apresentam nível 5; e outros quatro estudos trazem nível 6 de evidência, nestes últimos casos não há fortes evidências para aplicação clínica.

Os artigos analisados ensejaram dados atuais acerca da prevenção e tratamento da UP. Em decorrência dos níveis de evidências e dos conteúdos das pesquisas publicadas, os artigos foram agrupados em duas categorias que emergiram das informações adquiridas: 1estudos que objetivaram descrever os fatores de risco para o desenvolvimento das UP, bem como as estratégias de prevenção e o tratamento empregado; 2- estudos que descreveram o impacto de intervenções educativas, o desempenho organizacional das equipes e qualidade no atendimento. Cinco artigos foram incluídos na primeira categoria e oito na segunda, como será possível observar na discussão dos resultados.

\section{Categoria 1 - Fatores de risco, estratégias de prevenção e tratamento para úlcera por pressão}


Nesta categoria, o estudo de Salcido, Lee e Ahn (2011) relata que a prevenção eficaz de UP no calcanhar envolve uma abordagem multifacetada, incluindo identificação de riscos, avaliação de comorbidades e implementação de dispositivos de redistribuição de pressão precocemente. Salienta que deve haver correta distinção de doenças, como a síndrome do calcanhar roxo e a lesão profunda dos tecidos que iminentemente causam isquemia e necrose dos tecidos, podendo representar fator de confusão com a UP.

Conforme Chou et al. (2013), superfícies de apoio estático mais sofisticados são mais eficazes do que colchões-padrão para prevenir úlceras nas populações de maior risco, corroborando o estudo de Pham et al. (2011), que salienta que a evidência clínica e econômica suporta o uso de colchões de redistribuição de pressão para todos os residentes que estão em cuidados de longo prazo.

Pham et al. (2011) retratam que a prevenção com produtos de limpeza de espuma perineais e o uso de emolientes para pele seca parecem ser custo efetivo, mas conclusões firmes são limitadas pela evidência clínica disponível. Chou et al. (2013) corroboram com Pham et al. (2011) quando relatam evidências sobre a eficácia da suplementação nutricional, reposicionamento e as intervenções de cuidados da pele, acrescentando que o cuidado usual ainda é limitado e contém falhas metodológicas, impedindo boas conclusões.

Conforme Anders et al. (2010), a aplicação e a eficácia das medidas de prevenção da UP devem ser reavaliadas e documentadas. Uma vez que os riscos foram avaliados, devem ser tomadas medidas terapêuticas com base no perfil de risco individual do paciente, com ênfase no encorajamento ativo do movimento e alívio passivo da pressão por mudanças frequentes de posição. Para Sullivan e Schoelles (2013), a integração de vários componentes na melhoria dos processos de atendimento reduz as taxas de UP, incluindo a simplificação e a padronização de intervenções específicas para prevenir UP, a documentação destas, o envolvimento de equipes multidisciplinares e a formação de especialistas designados para avaliação da pele por meio de cursos de educação de funcionários, auditorias e feedback.

Constatou-se que seriam necessárias mais pesquisas para determinar se intervenções de cuidados - reposicionamento, nutricionais ou de pele - mais intensas são mais eficazes do que os cuidados preventivos ordinários - incluindo padrão de reposicionamento, nutricionais e cuidados com a pele. Embora as pesquisas evidenciem que todos os cuidados - intervencionais ou preventivos - são pertinentes para evitar ou tratar as UP, os estudos em torno dessa temática, no entanto, devem preocupar-se em relatar o risco basal de UP em pacientes avaliados e considerar as análises de subgrupos predefinidos para ajudar na compreensão dos profissionais e familiares cuidadores acerca da maneira como as intervenções preventivas podem ser desenvolvidas (Chouet al., 2013).

\section{Categoria 2 - Estratégias educativas,desempenho organizacional das equipes e qualidade no atendimento.}

Conforme estudo de Figueiredo et al. (2010), as famílias e cuidadores não têm conhecimento adequado sobre medidas de prevenção sobre UP, o que torna essencial investir em ações avaliativas e educacionais para ampliar o conhecimento de membros familiares e cuidadores sobre ações corretas a serem desenvolvidas para a prevenção de UP. Já estudo de Saunders, Krause e Reed (2010) deflagra que a falta de recursos, tanto financeiros como 
educacionais, está associada a piores resultados das UP, agravadas entre as pessoas com lesão medular espinhal.

McGuinnesset al. (2012) inferem que a introdução de equipes especialistas para avaliar rotineiramente a pele de pacientes, seja em unidade de neurologia, neurocirurgia, cardiovascular, reabilitação ou unidades médicas/pós-operatório outras, parece ser um método eficaz para reduzir a UP. Os atos de educar e motivar os profissionais da enfermagem e auxiliares desta área para proporcionar cuidado com excelência com a pele dos pacientes possibilitam limitar/eliminar a UP adquirida em hospital. Picatoste et al.(2012) confirmam a eficácia das ações educativas em Unidade de Terapia Intensiva (UTI) cirúrgica na redução da incidência de UP em seu estudo e, inclusive, demonstram que promover capacitação profissional com a enfermagem melhora e minimiza os registros de UP.

Rantz et al. (2012) lecionam que, além dos cuidados restritos ao ambiente hospitalar, a intervenção com enfoque na qualidade do cuidado em lares de idosos demonstrou melhora significativa na qualidade do atendimento, bem como nos índices de UP. Dahlstrom et al. (2011) asseveram o exposto e relatam que, para manter o sucesso e melhoria da qualidade na prevenção e tratamento de UP, são de fundamental importância as intervenções frequentes e repetidas, independentemente do local onde o paciente se encontra - domicílio ou hospital.

Segundo Lee, Kim e Cho (2011), para manter e melhorar a atividade de vida diária de idosos internados em hospitais, demandando cuidados a longo prazo, deve-se desenvolver estratégias para prevenir a incontinência urinária e fecal, UP, tubos ou cateteres desnecessários, oferecendo fisioterapia adequada. Palma e Sánchez (2010) acrescentam que a adoção e implementação de orientações de boas práticas para a prevenção e tratamento de UP oferecem elevada variabilidade como obstáculos no plano de gestão e instituições, custos econômicos e materiais, treinamento, atitudes, motivação pessoal e implementação de cuidados.

Os pesquisadores ora mencionados - Figueiredo et al. (2010); Saunders,Krause e Reed (2010); Palma e Sánchez (2010); Lee, Kim e Cho (2011); Dahlstromet al.(2011); Picatoste et al. (2012); McGuinnesset al. (2012) - são congruentes no tocante às recomendações para a melhora dos cuidados de prevenção e tratamento UP: utilização de protocolos adaptados; o compromisso da instituição em implementar e fiscalizar a realização das ações descritas no protocolo; formação interdisciplinar dos profissionais de saúde e dos cuidadores e avaliação contínua do paciente.

Em razão da magnitude do problema da UP, considerado evento adverso, é um dos seis eixos prioritários de monitoramento e prevenção no Programa Nacional de Segurança do Paciente, desde 2013, no Brasil. Com base nessa premissa, a Agência Nacional de Vigilância Sanitária (Anvisa) estabeleceu a obrigatoriedade da criação de núcleos de segurança do paciente em todos os serviços de saúde públicos e privados, e tornou compulsória a notificação da UP, sendo esta considerada incidente que resulta em dano ao paciente. Neste sentido, este ensaio vem ao encontro do propósito das metas de segurança do paciente, que tem como foco a prevenção da UP, direcionando medidas para esta garantia. 


\section{Considerações finais}

A revisão integrativa possibilitou constatar que não há uma vasta produção científica veiculada à temática, o que enseja a necessidade de maior atenção a pesquisas nessa área. $A$ elaboração de uma síntese do conhecimento científico acerca das UP, no concernente às medidas de prevenção primária e de educação, na perspectiva da literatura atual, ainda que em parcos artigos, no entanto, permitiu averiguar a importância do conhecimento para a prevenção e tratamento das UP, fato sinalizador de que este estudo pode contribuir no âmbito da educação em saúde.

No que tange à utilização de medidas preventivas, pesquisadores - Salcido, Lee eAhn (2011);Chou et al. (2013); Pham et al. (2011); Anders et al. (2010); Sullivan eSchoelles (2013) demonstram em seus estudos que enquanto algumas apresentam efeitos benéficos comprovados e com bom nível de evidência, outras medidas parecem não exprimir efeitos suficientemente comprovados como benéficos ou eficazes. Em relação às medidas educativas, estudos de Figueiredo et al. (2010); Saunders, Krause e Reed (2010); McGuinnesset al. (2012); Picatoste et al. (2012); Rantz et al. (2012);Dahlstromet al.(2011); Lee, Kim e Cho (2011); Palma e Sánchez (2010), unanimemente, revelam resultados benéficos, embora alguns não representem dados estatisticamente significativos, os pesquisadores consideram os resultados importantes, porque envolvem melhoria considerável da qualidade de vida do paciente. A educação foi considerada um dos pilares de sucesso no tratamento de UP, contudo, autores enfatizam a importância de intervenções educativas frequentes e repetidas para manter o sucesso e melhoria da qualidade na prevenção e tratamento das úlceras.

Observou-se que o impacto de intervenções educativas, o desempenho organizacional das equipes e qualidade no atendimento, o conhecimento acerca dos fatores de risco para o desenvolvimento das UP, bem como as estratégias de prevenção e o tratamento empregado, são os assuntos mais enfatizados nas pesquisas. Constata-se, no entanto, a necessidade de mais estudos que avaliem a prevenção primária associada a medidas educativas em vários contextos de atendimento, como hospitais, instituições de longa permanência, UTIs e no âmbito domiciliar, analisando informações sobre condições específicas de cada paciente e do ambiente de trabalho, bem como do processo de trabalho das equipes e da visão dos gestores responsáveis pelas instituições em relação às UP - para possibilitar entender melhor como as intervenções preventivas podem ser direcionadas.

A carência de estudos que abordem equipes multidisciplinares pertinentes à prevenção primária e à educação no que respeita às UP não condiz com a importância da temática, pois, a origem da UP é multifatorial e a sua prevenção e o tratamento devem envolver toda a equipe de saúde. Programas educacionais há de estar disponíveis a todos os níveis de cuidadores, pacientes e seus componentes familiares, no entanto, somente as equipes de enfermagem é que, geralmente, são avaliadas quanto às medidas preventivas e participam de intervenções educativas na maioria dos estudos. 


\section{REFERÊNCIAS}

Anders, J., Heinemann, A., Leffmann, C., Leutenegger, M., Pröfener, F. \& von Renteln-Kruse, W. (2010).Decubitus ulcers: pathophysiology and primary prevention. Deutsches Arzteblatt International,107(21), 371-382.

Baron, M. V., Carneiro, M.,Fialho, L. M. F. \&Santana, J. R.(2012). Úlceras por pressão: uma abordagem multidisciplinar. Fortaleza: UFC.

Bento, A. (2012). Como fazer uma revisão da literatura: considerações teóricas e práticas. Revista JA, 7(65), 42-44.

Chou, R., Dana, T., Bougatsos, C., Blazina, I., Starmer, A. J., Reitel, K. \& Buckley, D. I. (2013).Pressure ulcer risk assessment and prevention: a systematic comparative effectiveness review.Annals of Internal Medicine, 159(1), 28-38.

Dahlstrom, M., Best, T., Baker, C., Doeing, D., Davis, A., Doty, J. \& Arora V. M. (2011).Improving identification and documentation of pressure ulcers at an urban academic hospital. Joint Commission Journal on Quality and Patient Safety,37(3), 123-130.

Dardengo, D. D. et al. (2006). Avaliação do índice de conhecimento dos cuidadores de clientes portadores de úlcera por pressão em um bairro do município de Cachoeiro de Itapemirim. Revista Camiliana de Iniciação Científica, 1(1).

European Pressure Ulcer Advisory Panel and National Pressure Ulcer Advisory Panel (EpuapNpuap). (2009). Prevention and treatment of pressure ulcers: quick reference guide. Washington.

Figueiredo, Z. M., Tirado, J. J., Mulet, F. V., Núñez, A. J., Andrade, L. M., Miranda, M. C.\&Monteiro, M. G. S. (2010). Úlceras por presión en personas con lesión medular: conocimiento de familiares y cuidadores. Avances en Enfermería,28(n. esp.), 29-38.

Galvão, C. M.,Sawada, N. O. \& Mendes, I. A. C. (2003).A busca das melhores evidências.Revista da Escola de Enfermagem da USP, 37(4), 43-50.

Ganong, L. H. (1987).Integrative reviews of nursing research. Research in Nursing and Health, 10(1), 1-11.

Hess, C. T. (2002)Tratamento de feridas e úlceras. (4a ed.).Rio de Janeiro: Reichmann \& Affonso.

Lee, J. Y.,Kim, E. Y.\& Cho, E. (2011).Factors impacting the physical function of older adults in Korean long-term care hospitals. Journal of Korean Academy of Nursing,41(6), 780-787.

Leite, N. P.,Sanches, A. J. S.\&Santiago, S. N. (2010). Úlceras por pressão:visão nutricional. In W. Malagutti\& C. T.Kakihara. Curativo, estomias e dermatologia: uma abordagem multiprofissional. (pp. 385-400). São Paulo: Martinari.

McGuinness, J., Persaud-Roberts, S., Marra, S., Ramos, J., Toscano, D.,Policastro, L.\&Epstein, N. E. (2012). How to reduce hospital-acquired pressure ulcers on a neuroscience unit with a skin and wound assessment team.Surgical Neurology International, 3, 138-143.

Melnyk, B. M. \& Fineout-Overholt, E. (2005). Making the case for evidence-based practice. In B. M. Melnyk \& E. Fineout-Overholt. Evidence-based practice in nursing \& healthcare:a guide to best practice. (pp. 3-24). Philadelphia: Lippincot Williams \& Wilkins. 
Miranda, L. A.,Ramos, R. S.,Oliveira, O. V. S.,Ramos, L. T.,Spezani, R. S.\&Souza, R. M. (2012). Ações de enfermagem na prevenção de úlcera por pressão em pacientes internados em unidades de clínica médica. Revista Enfermagem Atual in Derme,63,7-13.

Palma, M. R.\&Sánchez, J. M. V. (2010).La gestión de úlceras por presión en residencias de mayores: obstáculos y facilitadores. Gerokomos, 21(3), 108-113.

Pham, B., Stern, A., Chen, W., Sander, B., John-Baptiste, A., Thein, H. H., Gomes, T., Wodchis, W. P., Bayoumi, A., Machado, M., Carcone, S. \&Krahn, M. (2011).Preventing pressure ulcers in long-term care: a cost-effectiveness analysis.JAMAlnternal Medicine, 171(20), 18391847.

Paranhos, W. Y. (2003). Úlceras de pressão. In S. A. Jorge\& S. R. P. E.Dantas. Abordagem multiprofissional do tratamento de feridas. (pp. 287-298). São Paulo: Atheneu.

Picatoste, J. W., Barreira, Á. S., Pestonit, T. M., Villar, S. R., Baña, Á. P., Álvarez, M. \& Gestal, A. E. (2012).Efectividad de una intervención formativa en prevención de úlceras por presión en una unidad de cuidados intensivos quirúrgica: un estudio cuasi experimental.Gerokomos, 23(3), 128-131.

Rantz, M. J., Zwygart-Stauffacher, M., Hicks, L., Mehr, D., Flesner, M., Petroski, G. F., Madsen, R. W., Scott-Cawiezell, J.(2012). Randomized multilevel intervention to improve outcomes of residents in nursing homes in need of improvement.Journal of the American Medical Directors Association, 13(1), 60-68.

Rocha, J. A.,Miranda, M. J.\&Andrade, M. J. (2006). Abordagem terapêutica das úlceras de pressão: intervenções baseadas na evidência. Acta Médica Portuguesa, 19, 29-38.

Salcido, R.,Lee, A. \& Ahn, C. (2011). Heel pressure ulcers: purple heel and deep tissue injury.Advances in Skin \&Wound Care, 24(8), 374-380.

Saunders, L. L.,Krause, J. S.\&Reed, K. S. (2010).The relationship of pressure ulcers, race, and socioeconomic conditions after spinal cord injury.The Journal of Spinal Cord Medicine,33(4), 387-395.

Sullivan, N. \& Schoelles, K. M. (2013).Preventing in-facility pressure ulcers as a patient safety strategy: a systematic review.Annals of Internal Medicine,158(5), 410-416.

Whittemore,R. \& Knafl, K. (2005). The integrative review: updated methodology. Journal of Advanced Nursing, 52(5), 546-553. 